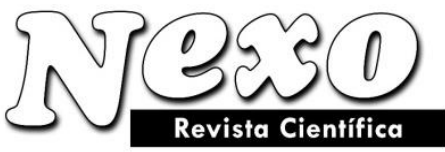

\title{
Efecto de la cocción en el contenido de compuestos bioactivos en quinua (Chenopodium quinoa Willd) de diferentes variedades de Perú
}

\section{Effect of cooking on the content of bioactive compounds in quinoa (Chenopodium quinoa Willd) of different varieties of Peru}

\author{
Zanhy L. Valencia-Reyes ${ }^{1 *(D)}$, Fiorella Vanessa Güere Salazar ${ }^{1}$ (D), Eduardo Zorrilla- \\ Tarazona $^{2}(\mathrm{D})$, Doris Fuster-Guillen ${ }^{1}(\mathrm{D})$, Jacinto Joaquín Vertiz-Osores ${ }^{3}$ (D) \\ ${ }^{1}$ Universidad Nacional Mayor de San Marcos, Lima, Perú \\ ${ }^{2}$ Universidad Nacional Autónoma de Huanta, Ayacucho, Perú \\ ${ }^{3}$ Universidad Nacional Tecnológica de Lima Sur, Lima, Perú \\ * Corresponding author E-mail: zvalenciar@unmsm.edu.pe
}

(recibido/received: 28-agosto-2021; aceptado/accepted: 15-septiembre-2021)

\section{RESUMEN}

El objetivo fue determinar la variabilidad de la concentración de compuestos bioactivos en seis muestras de quinua de Perú. Se evaluaron las variedades 'Blanca de Junín', 'Amarillo Marangani', 'Pasankalla', 'Altiplano Lima', 'Negra Collana', 'Altiplano Puno', las cuales fueron proporcionadas por el INIA. Las muestras seleccionadas se cocinaron $(250 \mathrm{~g} / 500 \mathrm{ml}$ agua destilada) durante $30 \mathrm{~min}$. Se observaron diferencias significativas por variedad, condición e intersección de ambos usando el análisis de varianza. Con respecto al contenido de compuestos fenólicos se notó diferencia significativa por variedad: 'Amarillo Marangani' tuvo el máximo promedio (2.1287 GAE mg/g). Hubo diferencia significativa de actividad antioxidante por variedad: 'Negra Collana' tuvo el promedio máximo $(0.5161 \mathrm{mg}$ GAE/g), También hubo diferencia significativa de flavonoides por variedad: 'Altiplano Puno' tuvo el promedio máximo $(0.6439 \mathrm{mg} / \mathrm{g})$. Para las betacianinas hubo diferencia significativa por variedad y condición. 'Altiplano Puno' tuvo un máximo promedio (0.5717 $\mathrm{mg} / 100 \mathrm{~g}$ ), siendo diferente por variedad, condición y ambos. Por último, 'Altiplano Puno' tuvo el promedio más alto de betaxantinas $(2.1125 \mathrm{mg} / 100 \mathrm{~g})$. Finalmente se tiene evidencia para afirmar que la cocción tuvo efecto en el contenido de compuestos bioactivos siendo significativamente diferente en las variedades de quinua evaluadas.

Palabras Clave: Compuestos fenólicos; actividad antioxidante; flavonoides; betacianinas; betaxantinas.

\section{ABSTRACT}

The objective was to determine the variability of the concentration of bioactive compounds in six samples of quinoa from Peru. The varieties 'Blanca de Junin', 'Amarillo Marangani', 'Pasankalla', 
'Altiplano Lima', 'Negra Collana', 'Altiplano Puno', which were provided by INIA, were evaluated. The selected samples were cooked $(250 \mathrm{~g} / 500 \mathrm{ml}$ of distilled water) for $30 \mathrm{~min}$. Significant differences were observed by variety, condition, and the intersection of both by analysis of variance. Regarding the content of phenolic compounds, a significant difference was noted by variety: 'Amarillo Marangani' had the maximum average $(2.1287 \mathrm{GAE} \mathrm{mg} / \mathrm{g})$. There was a significant difference in antioxidant activity by variety: 'Negra Collana' had the maximum average $(0.5161 \mathrm{mg}$ GAE/g). There was also a significant difference in flavonoids by variety: 'Altiplano Puno' had the maximum average $(0.6439 \mathrm{mg} / \mathrm{g})$. For betacyanins there was a significant difference by variety and condition. 'Altiplano Puno' had a maximum average $(0.5717 \mathrm{mg} / 100 \mathrm{~g})$, being different by variety, condition and both. Finally, 'Altiplano Puno' had the highest betaxanthin average $(2.1125 \mathrm{mg} / 100 \mathrm{~g})$. Finally, there is evidence to affirm that cooking had an effect on the content of bioactive compounds, being significantly different in the evaluated varieties of quinoa.

Keywords: Phenolic compounds; antioxidant activity; flavonoids; betacyanins; betaxanthins.

\section{INTRODUCCIÓN}

Se entiende por quinua a los granos de Chenopodium quinoa Willd (FAO, 2019). Esta planta se adapta bien a un rango de altitudes desde los 500-4.000 m. s. n. m., resultando ser un cultivo menos sensible a la salinidad, al estrés por la sequía y a las heladas, siendo parámetros que se deben establecer puntualmente para cada variedad. Este producto alimenticio constituye una importante alternativa para la implementación de políticas y planes de desarrollo gubernamentales, principalmente en países en desarrollo, pudiendo dirigirlos a reducir los latentes índices de malnutrición (Chito-Trujillo et al., 2017). En Perú, debido a su posición como el mayor productor de quinua del mundo, han surgido preguntas sobre la medida en que la expansión de la demanda mundial del cultivo es compatible con una gestión adecuada de los recursos naturales(Bedoya-Perales et al., 2018), sin embargo, para responder a estos cuestionamientos, se hace imprescindible la investigación en muchos aspectos más sobre este cultivo.

La quinua es un cultivo interesante, sus propiedades nutricionales y resistentes al medio ambiente requieren una mayor pesquisa en todos los campos de la biología vegetal, la agronomía, la ecología y ciencia y tecnología de los alimentos(Romano and Ferranti, 2019). Este vegetal ha sido seleccionado por la FAO como uno de los cultivos destinados a ofrecer seguridad alimentaria en el próximo siglo (FAO, 2019) pues es un alimento altamente funcional ya que posee muchos componentes que son importante en términos de salud humana (Ceyhun-Sezgin and Sanlier, 2019).

Este alimento es valioso por su buena composición en compuestos bioactivos (Pereira et al., 2020), sugiriéndose un particular interés por el estudio del efecto de la cocción sobre la concentración de estos compuestos (Lalaleo et al., 2020), pues, el modo de consumo humano más conocido es a través de la cocción. No obstante, hay evidencias que este proceso físico tiene influencia sobre la capacidad antioxidante en este alimento, pudiendo vincularse principalmente a su contenido de compuestos fenólicos y flavonoides (Dini et al., 2010), los mismos que, recientemente, están adquiriendo mayor interés por sus propiedades antioxidantes y funcionales, aunque solamente se han estudiado algunos de ellos quedando mucho más por hacer.

Por otra parte, debido a que este vegetal es oriundo de Perú, la diversidad natural de la especie es elevada, la misma que está vinculada a los microclimas que presenta este territorio multi-climático (Alandia et al., 2020; Covarrubias et al., 2020). En ese contexto, es importante el estudio de la 
composición de la quinua acorde con sus variedades, como previamente ha sido evidenciado en variedades introducidas en otros territorios (Covarrubias et al., 2020) pues esa información podría contribuir con la dilucidación de los mejores biotipos, pudiendo abrir opciones agro-ecológicas para un mayor cultivo de las mismas y posterior comercialización brindando mayores condiciones de desarrollo económico de los productores locales y, sobre todo, permitiéndose la incorporación de estas variedades en las dietas de las personas, especialmente de aquellas más vulnerables en términos de salud. Por tal el objetivo planteado fue evaluar el efecto de la cocción en el contenido de compuestos bioactivos en quinua de seis diferentes variedades de Perú.

\section{MÉTODOS}

\subsection{Muestras.}

Se recolectaron seis variedades de quinua en el Instituto Nacional de Innovación Agraria (INIA) provenientes de diferentes lugares del Perú; Blanca de Junín (Cusco), Amarillo Maranganí (Cusco), Pasankalla (Cusco), Altiplano Lima (Lima), Negra Collana (Puno), Altiplano Puno. (Puno). Las muestras se cocinaron hirviendo $250 \mathrm{~g}$ en $500 \mathrm{ml}$ agua destilada durante $30 \mathrm{~min}$.

\subsection{Procedimiento analítico.}

Composición proximal: Para el análisis de la composición proximal se utilizaron métodos normalizados ((AOAC, 2012) para las evaluaciones de humedad (930.04), proteínas (977.02) con algunas modificaciones (AOAC, 2012), y grasas mediante método Soxtec ${ }^{\mathrm{tm}} 2000$ (FOSS IBERIA, 2012).

\subsection{Preparación de los extractos}

Para los análisis de fenólicos totales, capacidad antioxidante, flavonoides y betalaínas se usaron extractos de EtOH- $\mathrm{H}_{2} \mathrm{O}$ (1:1), preparados de la siguiente manera: alrededor de $100 \mathrm{mg}$ de muestra molida $(\varnothing<0.5 \mathrm{~mm})$ se extraen con $10 \mathrm{~mL}$ de solución hidroalcohólica durante 12 horas a $4{ }^{\circ} \mathrm{C}$ por maceración, posteriormente los extractos se filtran a través de un filtro de membrana de $0.45 \mu \mathrm{m}$ para su análisis. Adicionalmente, $100 \mathrm{mg}$ de muestra molida se extrajeron con $5 \mathrm{~mL}$ de solución $\mathrm{MeOH}: \mathrm{H}_{2} \mathrm{O}(2: 1)$ durante 60 minutos a $50{ }^{\circ} \mathrm{C}$, los extractos se filtraron a través de una filtro de membrana de $0.45 \mu \mathrm{m}$ y se enrazaron a un volumen de $10 \mathrm{~mL}$ con la misma solución, para su análisis.

\subsection{Fenólicos totales}

Los fenólicos totales se determinaron de la siguiente manera $0.1 \mathrm{~mL}$ del extracto se mezclaron con $7.9 \mathrm{~mL} \mathrm{H}_{2} \mathrm{O}$ destilada y $0.5 \mathrm{~mL}$ del reactivo de Folin Ciocalteu, se agitó y agregó $1.5 \mathrm{~mL}$ de $\mathrm{Na}_{2} \mathrm{CO}_{3}$ al 20\%(Dini et al., 2010), la solución se dejó en reposo 2 h y al finalizar el tiempo se midió la absorbancia a $760 \mathrm{~nm}$ en el espectrofotómetro UV-Vis (SPEKOL 1500, Alemania). Los fenólicos totales se expresaron como equivalentes $\mathrm{mg}$ de ácido gálico GAE/g, a partir de una curva de calibración $\left(\mathrm{r}^{2}=0.9988, \mathrm{y}=0.0011 \mathrm{x}\right)$.

\subsection{Actividad antioxidante}

La capacidad antioxidante de las diferentes muestras se determinó de la siguiente manera 1,1-difenilo2picril-hidrazilo (DPPH), en resumen $0,3 \mathrm{~mL}$ de extracto hidroalcohólico (1:1) se mezcló con 2,7 mL de solución EtOH de DPPH (Fischer et al., 2013), se agitó y dejó en reposo por 30 min, al término de los cuales se midió la absorbancia a $517 \mathrm{~nm}$ en el espectrofotómetro UV-Vis (SPEKOL 1500, Alemania), la actividad antioxidante se reportó como equivalentes $\mathrm{mg}$ de ácido gálico (GAE)/g 
muestra, a partir de una curva de calibración del \% de inhibición vs ácido gálico $\left(\mathrm{r}^{2}=0.9909\right.$, $\mathrm{y}=5.4244 \mathrm{x})$.

\subsection{Flavonoides totales}

Los flavonoides totales se determinaron de la siguiente manera $0.5 \mathrm{~mL}$ del extracto EtOH- $\mathrm{H}_{2} \mathrm{O}(1: 1)$ de la muestra se mezcló con $2 \mathrm{~mL} \mathrm{H}_{2} \mathrm{O}$ destilada y $0.15 \mathrm{~mL}$ de $\mathrm{NaNO}_{2}(5 \mathrm{~g} / 100 \mathrm{ml})$, después de 5 min se agregó $0.15 \mathrm{~mL}$ de solución acuosa de $\mathrm{AlCl}_{3}(10 \mathrm{~g} / 100 \mathrm{~mL})$, y luego de 6 min se mezcló con $1 \mathrm{~mL}$ de $\mathrm{NaOH}(1 \mathrm{~N})$, finalmente la solución se enrazó con $1.2 \mathrm{~mL}$ de $\mathrm{H}_{2} \mathrm{O}$ destilada a $5 \mathrm{~mL}$, agitándose la solución (Dini et al., 2010). La concentración de flavonoides totales se determinó a partir de la absorbancia de la solución a $510 \mathrm{~nm}$ en el espectrofotómetro UV-Vis (SPEKOL 1500, Alemania) y estos se expresaron como mg equivalente de catequina/g de muestra, a partir de una curva de calibración $\left(\mathrm{r}^{2}=0.9995, \mathrm{y}=0.0032 \mathrm{x}\right)$.

\subsection{Betalaínas}

Las betalaínas se determinaron de la siguiente manera $0.2 \mathrm{~g}$ de la muestra se extrajeron con $10 \mathrm{~mL}$ de $0.05 \mathrm{M}$ de buffer fosfato a pH 6.5 (von Elbe, 2001), la mezcla se agitó $2 \mathrm{~h}$ a temperatura ambiente y oscuridad, al finalizar el tiempo señalado, las muestras se centrifugaron a $13000 \mathrm{rpm}$ a la temperatura de $5{ }^{\circ} \mathrm{C}$ por $30 \mathrm{~min}$, finalmente se miden las absorbancias de las muestras a 538, 476 y $600 \mathrm{~nm}$ (von Elbe, 2001) en el espectrofotómetro UV-Vis (SPEKOL 1500, Alemania). La determinación de betacianinas $\mathrm{y}$ betaxantinas resulta de reemplazar los valores de absorbancia en las expresiones: $\mathrm{x}=$ 1.095(a-c), y = b-z-x/3.1 y z = a-x (von Elbe, 2001); donde: a= absorbancia a $538 \mathrm{~nm}, \mathrm{~b}=$ absorbancia a $476 \mathrm{~nm}, \mathrm{c}=$ absorbancia a $600 \mathrm{~nm}, \mathrm{x}=$ absorbancia debido a betacianina, $\mathrm{y}=$ absorbancia debido a betaxantinas, $\mathrm{y} z=$ absorbancia debido a impurezas.

\section{8. $\quad$ Análisis de datos}

Los resultados se expresaron como media \pm desviación estándar de tres repeticiones. Posteriormente, los resultados se sometieron a análisis de varianza (ANOVA), que se utilizó para comparar medias; las diferencias se determinaron mediante la prueba de Tukey al 5\% de probabilidad, y las correlaciones entre variables se determinaron mediante el coeficiente de correlación de Pearson ( $\mathrm{p}<$ 0,001). Todos los análisis se realizaron utilizando el software GraphPad Prism® V.9 y SPSS® V.27.0.

\section{RESULTADOS}

\subsection{Contenido total de proteína y grasa.}

La calidad nutricional de un producto depende tanto de la cantidad como de la calidad de sus nutrientes. El contenido total de proteína y materia grasa fueron analizados tal como se muestra en la Tabla 1. Los valores encontrados de proteína en las muestras crudas analizadas variaron de (14.53$17.57 \%$ ) la de más alto contenido de proteína fue la variedad Altiplano Lima. Existen trabajos nacionales e internacionales que reportan el rango de proteínas en quinua varía entre (10.4-17.0\%) (Reyes, 2006). Las quinuas cocidas analizadas variaron de (8.50-15.21\%) siendo la de más alto contenido de proteína la variedad Negra Collana. Aunque inicialmente la variedad Altiplano Lima. fue la que obtuvo el más alto porcentaje de proteína, después de la cocción la proteína se desnaturalizó a causa del calor como ya está documentado (Avila-Ruiz et al., 2016), el procesamiento de quinuas dulces con calor puede influir en el valor nutritivo de la proteína de quinua (Roa et al., 2017). 
Tabla 1 . Contenido total de proteína $(\%$, b.s. ) y grasa $(\%$, b.s.) en quinua (media \pm desviación estándar, $n=3$ )

\begin{tabular}{cccc}
\hline \multirow{2}{*}{$\begin{array}{c}\text { Variedades de } \\
\text { Quinua }\end{array}$} & \multicolumn{2}{c}{ proteína $(\%$, b.s. $)$} & grasa $(\%$, b.s. $)$ \\
\cline { 2 - 4 } & Quinua cruda. & Quinua cocinada & Quinua cruda \\
\hline $\begin{array}{c}\text { Blanca de Junín } \\
\text { Amarillo }\end{array}$ & $16.20 \pm 0.00$ & $11.07 \pm 0.28$ & $2.13 \pm 1.65$ \\
\hline Maranganí & $16.10 \pm 0.13$ & $8.50 \pm 0.56$ & $0.33 \pm 0.25$ \\
\hline Pasankalla & $17.08 \pm 0.14$ & $12.65 \pm 0.28$ & $0.67 \pm 0.49$ \\
\hline Altiplano Lima & $17.57 \pm 0.56$ & $14.83 \pm 0.55$ & $0.70 \pm 0.30$ \\
\hline Negra Collana & $17.08 \pm 0.14$ & $15.21 \pm 0.01$ & $4.30 \pm 0.53$ \\
\hline Altiplano Puno & $14.53 \pm 1.27$ & $13.54 \pm 0.14$ & $5.17 \pm 0.67$ \\
\hline
\end{tabular}

El contenido de grasa en quinua sólo se analizó en muestras crudas por ser su contenido muy bajo variando de (0.33-5.17\%) siendo la variedad Altiplano Puno la que presentó mayor contenido. Existen valores de contenido de grasa en quinua reportado por la FAO de 555 accesiones de la colección boliviana de quinua de (2.05-10.88\%) (FAO, 2011), habiéndose también reportaron valores de $(2,05$ a $10,88 \%$ ) en 15 variedades de quinua boliviana (Rojas et al., 2016), por lo que, en base a esto, se indica que el contenido hallado fue bajo. El contenido de grasa de la quinua tiene un alto valor debido a su alto porcentaje de ácidos poliinsaturados como el grupo predominante, estos son el ácido linoleico (C18:2 $\omega 6)$, ácido linolénico (C18:3 $\omega 3)$ y en los ácidos monoinsaturados se destaca el ácido oleico (C18:1 $\omega 9$ ). La presencia de ácido linolénico le confiere una mayor inestabilidad oxidativa (Castro, 2010).

\subsection{Contenido de fenólicos totales, actividad antioxidante, flavonoides y betalaínas.}

El contenido de fenólicos totales, capacidad antioxidante, flavonoides y betalaínas en quinua cruda y cocida analizada se muestra en la Tabla 2. El contenido de compuestos fenólicos en las quinuas crudas varió de (1.25-2.48 GAE mg/g) el valor más alto lo tuvo la quinua Negra Collana, este valor es más bajo que lo descrito por Tang et al. (2015) quienes reportaron un contenido de fenólicos más altos en quinua negra (5.18 GAE mg/g). El contenido de compuestos fenólicos en las quinuas cocidas varió de (1.24-2.21 GAE mg/g) el valor más alto correspondió a la quinua Amarillo Maranganí. El contenido de compuestos fenólicos disminuyó durante la cocción como ya está bien documentado por Dini et al. (2010) quienes reportaron que los valores en quinua dulce disminuyeron de (7.72 a $2.87 \mathrm{GAE} \mathrm{mg} / \mathrm{g}$ ) y en quinua amarga de (8.64 a $5.94 \mathrm{GAE} \mathrm{mg} / \mathrm{g})$. Los valores encontrados en la presente investigación fueron mayores a los encontrados por Miranda et al. (2011) en quinuas cultivadas en tres zonas de producción de Chile $(0.14$ a $0.65 \mathrm{GAE} \mathrm{mg} / \mathrm{g})$ para seis ecotipos.

Tabla 2. Contenido de fenólicos totales (GAE mg/g, b.s.), actividad antioxidante según DPPH (mg equivalente de ácido gálico GAE/100g, b.s.), flavonoides (CAE mg/g, b.s.), betalaínas (mg/100 g) en muestras de quinua (media \pm desviación estándar, $n=3$ ) 


\begin{tabular}{|c|c|c|c|c|c|c|c|c|c|c|}
\hline \multirow{3}{*}{ Variedad } & \multirow{2}{*}{\multicolumn{2}{|c|}{$\begin{array}{l}\text { Compuestos } \\
\text { fenólicos totales } \\
\text { (GAE mg/g, b.s.) }\end{array}$}} & \multirow{2}{*}{\multicolumn{2}{|c|}{$\begin{array}{c}\text { Actividad } \\
\text { antioxidante DPPH } \\
\text { (mg eq de ácido } \\
\text { gálico GAE / } 100 \mathrm{~g}, \\
\text { b.s.) }\end{array}$}} & \multirow{2}{*}{\multicolumn{2}{|c|}{$\begin{array}{c}\text { Flavonoides } \\
\text { (CAE mg/g, b.s.) }\end{array}$}} & \multicolumn{4}{|c|}{ Betalaínas } \\
\hline & & & & & & & \multicolumn{2}{|c|}{$\begin{array}{c}\text { Betacianinas } \\
(\mathrm{mg} / 100 \mathrm{~g})\end{array}$} & \multicolumn{2}{|c|}{$\begin{array}{l}\text { Betaxantinas } \\
(\mathrm{mg} / 100 \mathrm{~g})\end{array}$} \\
\hline & $\begin{array}{l}\text { Quinua } \\
\text { cruda }\end{array}$ & $\begin{array}{l}\text { Quinua } \\
\text { cocida }\end{array}$ & $\begin{array}{l}\text { Quinua } \\
\text { cruda }\end{array}$ & $\begin{array}{l}\text { Quinua } \\
\text { cocida }\end{array}$ & $\begin{array}{l}\text { Quinua } \\
\text { cruda }\end{array}$ & $\begin{array}{l}\text { Quinua } \\
\text { cocida }\end{array}$ & $\begin{array}{l}\text { Quinua } \\
\text { cruda }\end{array}$ & $\begin{array}{l}\text { Quinua } \\
\text { cocida }\end{array}$ & $\begin{array}{l}\text { Quinua } \\
\text { cruda }\end{array}$ & $\begin{array}{l}\text { Quinua } \\
\text { cocida }\end{array}$ \\
\hline $\begin{array}{c}\text { Blanca de } \\
\text { Junín }\end{array}$ & $\begin{array}{c}1.25 \pm \\
0.06\end{array}$ & $\begin{array}{c}1.24 \pm \\
0.02\end{array}$ & $\begin{array}{c}44.93 \pm \\
0.01\end{array}$ & $\begin{array}{c}44.90 \pm \\
0.03\end{array}$ & $\begin{array}{c}0.39 \pm \\
0.02\end{array}$ & $\begin{array}{c}0.38 \pm \\
0.01\end{array}$ & $\begin{array}{c}0.49 \pm \\
0.07\end{array}$ & $\begin{array}{c}0.66 \pm \\
0.07\end{array}$ & $\begin{array}{c}1.20 \pm \\
0.22\end{array}$ & $\begin{array}{c}1.66 \pm \\
0.51\end{array}$ \\
\hline $\begin{array}{c}\text { Amarillo } \\
\text { Maranganí }\end{array}$ & $\begin{array}{c}2.24 \pm \\
0.39\end{array}$ & $\begin{array}{l}2.21 \pm \\
0.25\end{array}$ & $\begin{array}{c}53.62 \pm \\
0.00\end{array}$ & $\begin{array}{c}52.82 \pm \\
0.01\end{array}$ & $\begin{array}{c}0.56 \pm \\
0.13\end{array}$ & $\begin{array}{c}0.51 \pm \\
0.07\end{array}$ & $\begin{array}{c}0.48 \pm \\
0.06\end{array}$ & $\begin{array}{c}0.34 \pm \\
0.07\end{array}$ & $\begin{array}{c}1.50 \pm \\
0.11\end{array}$ & $\begin{array}{c}1.08 \pm \\
0.07\end{array}$ \\
\hline Pasankalla & $\begin{array}{c}1.93 \pm \\
0.22\end{array}$ & $\begin{array}{c}1.81 \pm \\
0.18\end{array}$ & $\begin{array}{c}50.16 \pm \\
0.00\end{array}$ & $\begin{array}{c}46.23 \pm \\
0.03\end{array}$ & $\begin{array}{c}0.61 \pm \\
0.01\end{array}$ & $\begin{array}{c}0.58 \pm \\
0.06\end{array}$ & $\begin{array}{c}0.43 \pm \\
0.04\end{array}$ & $\begin{array}{c}0.53 \pm \\
0.06\end{array}$ & $\begin{array}{c}1.15 \pm \\
0.07\end{array}$ & $\begin{array}{c}1.52 \pm \\
0.15\end{array}$ \\
\hline $\begin{array}{c}\text { Altiplano } \\
\text { Lima }\end{array}$ & $\begin{array}{c}1.92 \pm \\
0.22\end{array}$ & $\begin{array}{c}1.85 \pm \\
0.26\end{array}$ & $\begin{array}{c}36.70 \pm \\
0.00\end{array}$ & $\begin{array}{c}36.55 \pm \\
0.02\end{array}$ & $\begin{array}{l}0.40 \pm \\
0.05\end{array}$ & $\begin{array}{c}0.40 \pm \\
0.01\end{array}$ & $\begin{array}{c}0.35 \pm \\
0.02\end{array}$ & $\begin{array}{c}0.32 \pm \\
0.07\end{array}$ & $\begin{array}{c}0.92 \pm \\
0.06\end{array}$ & $\begin{array}{c}0.99 \pm \\
0.11\end{array}$ \\
\hline $\begin{array}{l}\text { Negra } \\
\text { Collana }\end{array}$ & $\begin{array}{c}2.48 \pm \\
0.30\end{array}$ & $\begin{array}{c}1.70 \pm \\
0.22\end{array}$ & $\begin{array}{c}56.98 \pm \\
0.01\end{array}$ & $\begin{array}{c}50.40 \pm \\
0.02\end{array}$ & $\begin{array}{c}0.56 \pm \\
0.01\end{array}$ & $\begin{array}{c}0.50 \pm \\
0.01\end{array}$ & $\begin{array}{c}0.49 \pm \\
0.24\end{array}$ & $\begin{array}{c}0.31 \pm \\
0.05\end{array}$ & $\begin{array}{c}1.01 \pm \\
0.28\end{array}$ & $\begin{array}{c}0.94 \pm \\
0.07\end{array}$ \\
\hline $\begin{array}{c}\text { Altiplano } \\
\text { Puno }\end{array}$ & $\begin{array}{c}1.71 \pm \\
0.27\end{array}$ & $\begin{array}{c}1.67 \pm \\
0.09\end{array}$ & $\begin{array}{c}35.77 \pm \\
0.01\end{array}$ & $\begin{array}{c}35.34 \pm \\
0.01\end{array}$ & $\begin{array}{c}0.69 \pm \\
0.02\end{array}$ & $\begin{array}{c}0.68 \pm \\
0.01\end{array}$ & $\begin{array}{c}0.41 \pm \\
0.03\end{array}$ & $\begin{array}{c}0.73 \pm \\
0.08\end{array}$ & $\begin{array}{c}0.94 \pm \\
0.04\end{array}$ & $\begin{array}{c}2.11 \pm \\
0.11\end{array}$ \\
\hline
\end{tabular}

El DPPH es un radical libre que puede obtenerse directamente sin una preparación previa, al evaluar la reacción entre los compuestos con actividad antioxidante y el radical estable 2,2-difenil1picrylhidrazilo (DPPH), se encontró un rango en las quinuas crudas de (35.77 a 56.98 GAE mg/100 $\mathrm{g})$, y en las quinuas cocidas de (35.34 a $52.8 \mathrm{GAE} \mathrm{mg/100} \mathrm{g),} \mathrm{la} \mathrm{actividad} \mathrm{antioxidante} \mathrm{disminuyó}$ durante la cocción a causa del incremento de temperatura como lo descrito por Dini et al. (2010) quienes reportaron que valores en quinua amarga y dulce disminuyeron casi a la mitad después de la cocción. Adicionalmente, Miranda et al. (2010) quienes indicaron que las muestras de quinua chilena disminuyeron su contenido de fenólicos totales y actividad antioxidante a causa del secado convectivo de 40 a $80{ }^{\circ} \mathrm{C}$, el efecto de altas temperaturas afecta el contenido inicial de los fenólicos totales y actividad antioxidante.

Por su parte, Valencia et al. (2017) reportaron valores en quinua cruda de 40,77 a 81,85 mg GAE/100 g. La correlación entre el contenido de fenólicos totales y actividad antioxidante sigue siendo un problema complejo debido a varios factores, tales como métodos de cocción, tipo de solvente de extracción y naturaleza de los fitoquímicos (Miranda et al., 2010).

El contenido de flavonoides en las quinuas crudas varió de (0.39-0.69 CAE mg/g), el valor más alto correspondió a la quinua Altiplano Puno. En las quinuas cocidas varió de $(0.38-0.68 \mathrm{CAE} \mathrm{mg/g).} \mathrm{Se}$ cree que los flavonoides en la dieta tienen beneficios para la salud, posiblemente debido a propiedades antioxidantes y antiinflamatorias. La quercetina es el antioxidante más potente entre los flavonoides. $\mathrm{Al}$ respecto, Hirose et al. (2010) reportaron que las semillas de quinua poseen grandes cantidades de quercetina, y las variedades cultivadas en Japón presentaron mayor cantidad que las cultivadas en América del Sur. Asimismo, Repo-Carrasco-Valencia et al. (2010) reportaron resultados en quinua de las zonas altoandinas de Perú, analizados por HPLC, el contenido de flavonoides fue excepcionalmente alto variando de 36.2 a $72.6 \mathrm{mg} / 100 \mathrm{~g}$, los flavonoides dominantes fue la quercetina y el Kaempferol, mientras que en algunas variedades se encontraron Myricetin e Isorhamnetin. En la 
Figura 1 se muestra el efecto de la cocción de seis variedades de quinua en el contenido de proteína, compuestos fenólicos, actividad antioxidante, flavonoides.
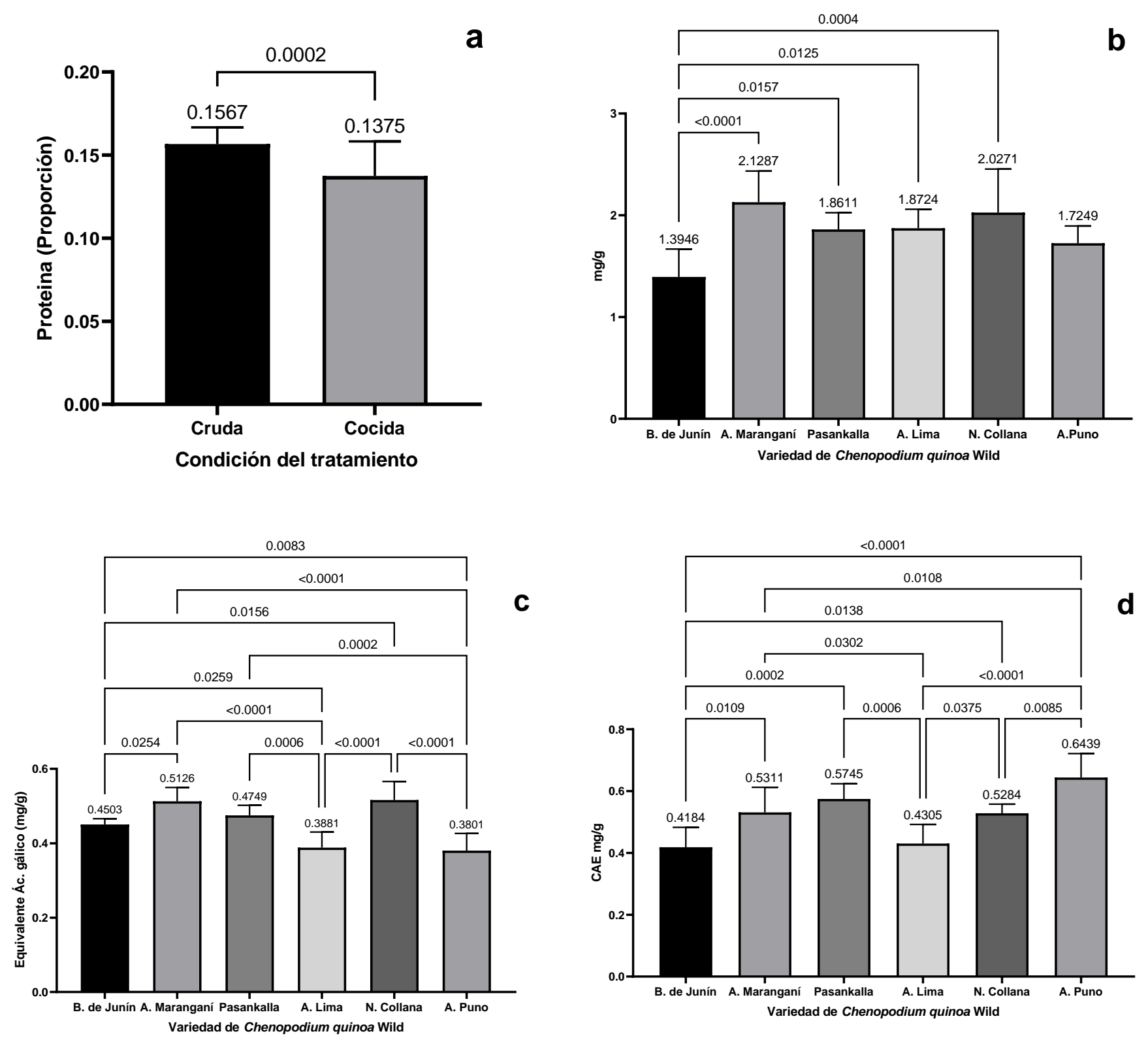

Figura 1. Efecto de la cocción de seis variedades de quinua en el contenido de: a) proteína, b) compuestos fenólicos, c) actividad antioxidante, d) flavonoides

Químicamente, las betalaínas son alcaloides derivados de la tirosina que pueden ser de dos tipos las betacianinas que son de color rojo-violáceo y las betaxantinas que son anaranjadas amarillentas, ambas con el núcleo fundamental del ácido betalámico. en la presente investigación se evaluó el contenido de betacianinas en las quinuas crudas de $(0.35-0.49 \mathrm{mg} / 100 \mathrm{~g})$, siendo las variedades con más alto contenido Blanca de Junín y Negra Collana, en las quinuas cocidas de (0.31-0.73 mg/100 g), siendo la variedad con más alto valor Altiplano Puno, el contenido de betaxantinas en quinuas 
crudas de (0.92-1.50 mg/100 g), siendo las variedades con más alto contenido Amarillo Maranganí, en las quinuas cocidas de $(0.99-2.11 \mathrm{mg} / 100 \mathrm{~g})$, siendo la variedad con más alto valor Altiplano Puno. En la Figura 2. Se muestra el efecto de la cocción de seis variedades de quinua en el contenido de betacianina y betaxantina.
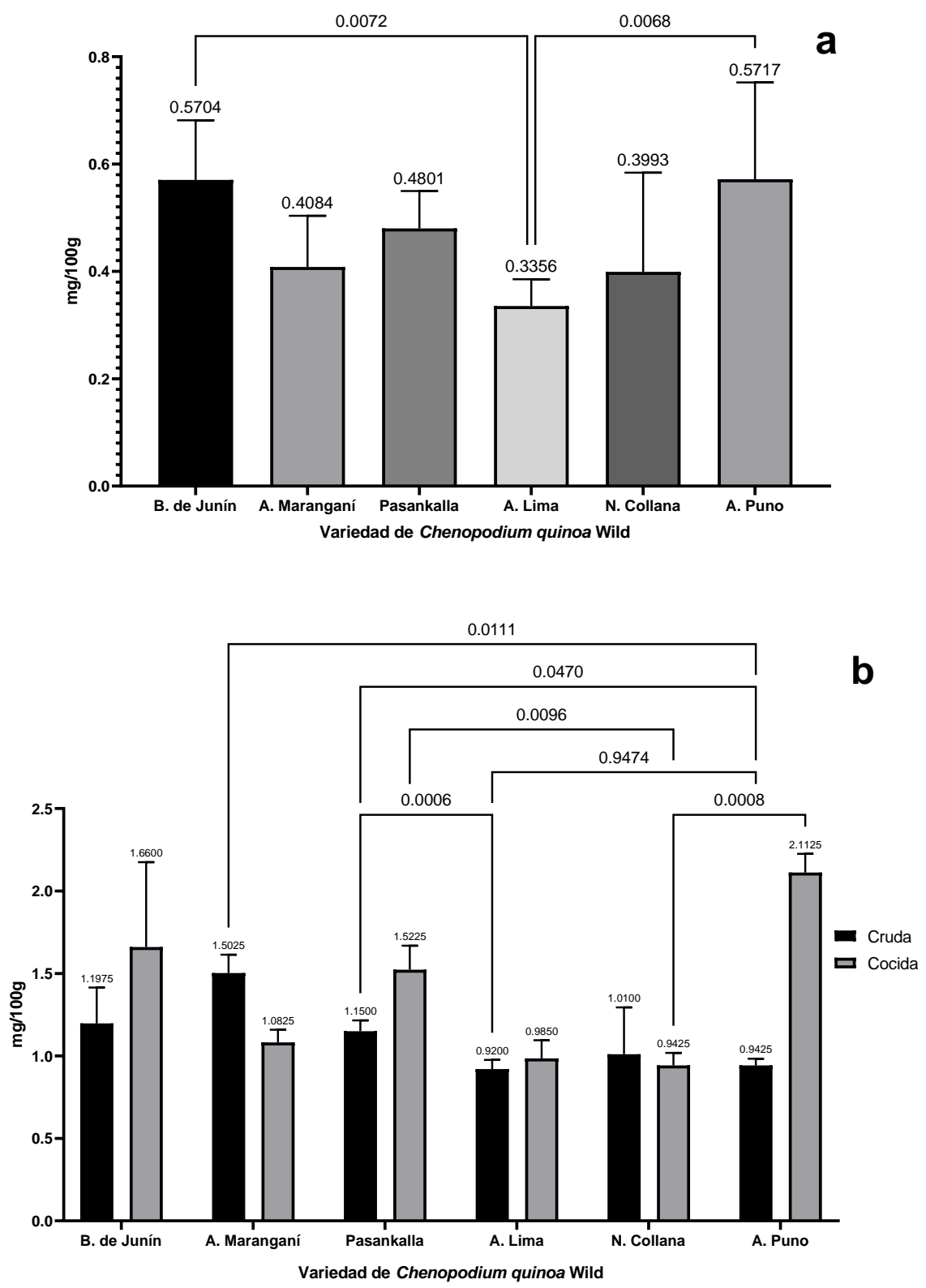

Figura 2. Efecto de la cocción de seis variedades de quinua en el contenido de a) betacianina, b) betaxantina

Si bien los resultados mostraron que el contenido de betacianinas y betaxantinas presentaron cantidades poco significativas, la muestra Altiplano Puno, es la que se mantuvo mejor con su contenido de éstos después de la cocción, además las muestras que fueron estudiadas carecían de colores intensos como amarillo o rojo. Esto coincide con lo reportado por Vidaurre-Ruiz et al. (2017) 
quienes reportaron que los pigmentos betalámicos en quinua Pasankalla y Negra Collana se degradaron difundiéndose en el agua de acción siguiendo una cinética de primer orden. La existencia de betaxantinas justifica el color exhibido en los granos amarillos y la mezcla con betacianinas genera la sombra roja (Escribano et al., 2017), por otra parte, existe poca información concerniente a betalaínas en quinua (Repo-Carrasco-Valencia et al., 2010).

\subsection{Contenido de proteína.}

\section{DISCUSIÓN}

Se observó diferencia significativa por condición (cruda o cocida) determinándose disminución del contenido de proteínas por el efecto de la cocción. La explicación es que la proteína se desnaturalizó a causa del calor tal como lo reportó Avila-Ruiz et al. (2016). Todas las variedades de quinuas crudas tienen más cantidad de proteína que las cocidas. Lo que permitiría optar por otros métodos de cocción que permitan mejor aprovechamiento de las unidades proteicas de este alimento, como por ejemplo los microondas (Wang et al., 2021) sin embargo, es una buena oportunidad para probar modos de cocción que permitan distinguir al que tenga menor efecto negativo sobre estas moléculas.

\subsection{Contenido de compuestos fenólicos.}

Se vio diferencia significativa por variedad, pero no por condición. Según lo reportado por Abderrahim et al. (2015), la cocción puede provocar un aumento de los compuestos fenólicos totales en muestras de quinua, sin embargo no explicitó si este aumento podría variar entre los biotipos de la quinua. El mayor valor con respecto a compuestos fenólicos lo presentó la variedad Amarillo Maranganí con un promedio de $2.1287 \mathrm{GAE} \mathrm{mg} / \mathrm{g}$, con una diferencia muy significativa en contraste con la variedad Blanca de Junín, que fue la variedad con mayor diferencia con todas las demás. Esto coincide con lo reportado por Abderrahim et al. (2015) quienes indicaron que el color de las distintas variedades de quinua tienen influencia sobre la cantidad inicial de compuestos fenólicos, aunque se conoce también que los colores de las variedades se vincular mucho a la composición química del suelo (Alandia et al., 2020), lo que explicaría la variabilidad de las concentraciones de fenólicas reportadas en este estudio.

\subsection{Actividad antioxidante.}

Se notó diferencia significativa por variedad, de manera indistinta existiendo el mismo comportamiento en variedades crudas y cocidas. Estadísticamente, las variedades Altiplano Puno y Altiplano Lima tuvieron similitud en actividad antioxidante, siendo la variedad Negra Collana la que mayores valores presentó con $0.5161 \mathrm{mg} \mathrm{GAE} / \mathrm{g}$. Según lo reportado por Nickel et al. (2016) una de las razones de la reducción de compuestos fenólicos y capacidad antioxidante en quinuas cocidos es el paso de compuestos al agua de cocción.

En referencia al contenido de flavonoides se observó diferencia significativa por variedad, pero no por condición. La cocción no influyó en el contenido de flavonoides. La variedad que presentó mayor contenido fue Altiplano Puno con $0.6439 \mathrm{mg} / \mathrm{g}$.

\subsection{Contenido de betacianinas}

Sí hubo diferencia significativa por variedad por lo que, la variedad si es diferente acorde a su condición de cruda o cocida. También existió diferencia significativa en la intersección de la variedad y condición. No existió diferencia significativa independiente por condición. El mejor promedio fue para la variedad Altiplano Puno que tuvo $0.5717 \mathrm{mg} / 100 \mathrm{~g}$. Con respecto al contenido de betaxantinas hubo diferencia significativa por variedad, condición y, por variedad y condición. El mejor promedio fue para la variedad Altiplano Puno con $2.1125 \mathrm{mg} / 100 \mathrm{~g}$. Finalmente, las semillas de quinua 
coloreada se proponen como una valiosa fuente natural de fenólicos, betalaínas con alta capacidad antioxidante (Abderrahim et al., 2015) pudiendo ser un referente para otros análisis comparativos a nivel internacional.

\section{CONCLUSIONES}

- El contenido de proteína presentó diferencia significativa por condición es decir que existe disminución en las proteínas por el efecto de la cocción.

- El contenido de compuestos fenólicos presentó diferencia significativa por variedad, pero no por condición. Para la actividad antioxidante presentó diferencia significativa sólo por variedad. El contenido de flavonoides presentó diferencia significativa por variedad, no por condición, la cocción no influyó en el contenido de flavonoides.

- El contenido de betacianinas presentó diferencia significativa por variedad, la variedad si es diferente cuando es cruda o cocida, también existe diferencia significativa por la intersección de variedad y condición. No hubo diferencia significativa por condición. El contenido de betaxantinas presentó diferencia por variedad, condición y variedad por condición, los resultados en el contenido total de betacianinas y betaxantinas mostraron cantidades poco significativas.

\section{AGRADECIMIENTOS}

Al Instituto Nacional de Innovación Agraria por permitir el uso de sus laboratorios para la realización experimental del presente trabajo, a la Universidad Nacional Tecnológica de Lima Sur por la capacitación mediante el Taller de Elaboración de Publicaciones Científicas.

\section{REFERENCIAS}

Abderrahim, F., Huanatico, E., Segura, R., Arribas, S., and Gonzalez, M. C. (2015). Physical features, phenolic compounds, betalains and total antioxidant capacity of coloured quinoa seeds (Chenopodium quinoa Willd.) from Peruvian Altiplano. Food Chemistry, 183, 83-90.

Alandia, G., Rodriguez, J. P., Jacobsen, S. E., Bazile, D., and Condori, B. (2020). Global expansion of quinoa and challenges for the Andean region. Global Food Security, 26, 100429. https://doi.org/10.1016/J.GFS.2020.100429

AOAC. (2012). Métodos Oficiales de Análisis. Association of Offical Analytical Chemistry. https://www.aoac.org/scientific-solutions/

Avila-Ruiz, G., Opazo-Navarrete, M., Meurs, M., Minor, M., Sala, G., van Boeke, M., Stieger, M., and Janssen, A. E. M. (2016). Denaturation and in Vitro Gastric Digestion of Heat-Treated Quinoa Protein Isolates Obtained at Various Extraction pH. Food Biophysics, 11, 184-197. https://doi.org/10.1007/s11483-016-9429-4

Bedoya-Perales, N. S., Pumi, G., Talamini, E., and Domingos Padula, A. (2018). The quinoa boom in Peru: Will land competition threaten sustainability in one of the cradles of agriculture? Land Use Policy, 79, 475-480.

Castro, E. S. (2010). Harina y aceite de quínoa (Quenopodium quinoa Willd.) de la región VI. 
https://bit.ly/3Ayx 7k2

Ceyhun-Sezgin, A., and Sanlier, N. (2019). A new generation plant for the conventional cuisine: Quinoa (Chenopodium quinoa Willd.). Trends in Food Science and Technology, 86, 51-58.

Chito-Trujillo, D. M., Ortega, R. A., Ahumada, A. F., and Rosero, B. (2017). Quinoa (Chenopodium quinoa Willd.) versus soja (Glycine max [L.] Merr.) en la nutrición humana: revisión sobre las características agroecológicas, de composición y tecnológicas. Revista Española de Nutrición Humana y Dietética, 21(2), 184-198.

Covarrubias, N., Sandoval, S., Vera, J., Núñez, C., Alfaro, C., and Lutz, M. (2020). Contenido de humedad, proteínas y minerales en diez variedades de quínoa chilena cultivadas en distintas zonas geográficas. Revista chilena de nutrición, 47(5), 730-737. https://doi.org/10.4067/s071775182020000500730

Dini, I., Tenore, G. C., and Dini, A. (2010). Antioxidant compound contents and antioxidant activity before and after cooking in sweet and bitter Chenopodium quinoa seeds. LWT - Food Science and Technology, $43,447-451$.

Escribano, J., Cabanes, J., Jiménez-Atiénzar, M., Ibañez-Tremolada, M., Gómez-Pando, L. R., GarcíaCarmona, F., and Gandía-Herrero, F. (2017). Characterization of betalains, saponins and antioxidant power in differently colored quinoa (Chenopodium quinoa) varieties. Food Chemistry, 234, 285-294.

FAO. (2011). La quinua: cultivo milenario para contribuir a la seguridad alimentaria mundial.

FAO. (2019). Codex Alimentarius. En Norma para la quinua (pp. 1-4). Food and Agriculture Organization.

Fischer, S., Wilckens, R., Jara, J., and Aranda, M. (2013). Variation in antioxidant capacity of quinoa (Chenopodium quinoa Will) subjected to drought stress. Industrial Crops and Products, 46, 341-349.

FOSS IBERIA, S. . (2012). Solución de extracción de disolventes de la serie Soxtec ${ }^{T M} 2000$ (pp. 1-10). Foss. https://bit.ly/3jIGNSv

Hirose, Y., Fujita, T., Ishii, T., and Ueno, N. (2010). Antioxidative properties and flavonoid composition of Chenopodium quinoa seeds cultivated in Japan. Food Chemistry, 119, 1300-1306.

Lalaleo, L., Hidalgo, D., Valle, M., Calero-Cáceres, W., Lamuela-Raventós, R. M., and Becerra-Martínez, E. (2020). Differentiating, evaluating, and classifying three quinoa ecotypes by washing, cooking and germination treatments, using 1 H NMR-based metabolomic approach. Food Chemistry, 331, 1-8.

Miranda, M., Vega-Gálvez, A., López, J., Parada, G., Sanders, M., Aranda, M., Uribe, E., and Di Scala, K. (2010). Impact of air-drying temperature on nutritional properties, total phenolic content and antioxidant capacity of quinoa seeds (Chenopodium quinoa Willd.). Industrial Crops and Products, 32, 258-263.

Miranda, M., Vega-Gálvez, A., Uribe, E., López, J., Martínez, E., Rodríguez, M. J., Quispe, I., and Di Scala, K. (2011). Physico-chemical analysis, antioxidant capacity and vitamins of six ecotypes of chilean quinoa (Chenopodium quinoa Willd). Procedia Food Science, 1, 1439-1446.

Nickel, J., Spanier, L. P., Botelho, F. T., Gularte, M. A., and Helbig, E. (2016). Effect of different types 
of processing on the total phenolic compound content, antioxidant capacity, and saponin content of Chenopodium quinoa Willd grains. Food Chemistry, 209, 139-143. https://doi.org/10.1016/j.foodchem.2016.04.031

Pereira, E., Cadavez, V., Barros, L., Encina-Zelada, C., Stojković, D., Sokovic, M., Calhelha, R. C., Gonzales-Barron, U., and Ferreira, I. C. F. R. (2020). Chenopodium quinoa Willd. (quinoa) grains: A good source of phenolic compounds. Food Research International, 137, 1-8.

Repo-Carrasco-Valencia, R., Hellström, J. K., Pihlava, J.-M., and Mattila, P. H. (2010). Flavonoids and other phenolic compounds in Andean indigenous grains: Quinoa (Chenopodium quinoa), kañiwa (Chenopodium pallidicaule) and kiwicha (Amaranthus caudatus). Food Chemistry, 120, 128-133.

Reyes, E. A. (2006). Componente nutricional de diferentes variedades de quinua de la región andina. AVANCES Investigación en Ingeniería, 5, 86-97.

Roa, D., Ortíz, V., and Tolaba, M. (2017). Comportamiento reológico de la fracción proteica del grano de amaranto extraída mediante molienda húmedo-ácida. Biotecnología en el Sector Agropecuario y Agroindustrial, 15, 123-130.

Rojas, W., Vargas Mena, A., and Pinto Porcel, M. (2016). La diversidad genética de la quinua: potenciales usos en el mejoramiento y agroindustria. RIIARn, 3(2), 114-124.

Romano, A., and Ferranti, P. (2019). Sustainable Crops for Food Security: Quinoa (Chenopodium quinoa Willd.). En Encyclopedia of Food Security and Sustainability (pp. 399-401). Elsevier Inc. https://doi.org/10.1016/B978-0-08-100596-5.22573-0

Tang, Y., Li, X., Zhang, B., Chen, P. X., Liu, R., and Tsao, R. (2015). Characterisation of phenolics, betanins and antioxidant activities in seeds of three Chenopodium quinoa Willd. genotypes. Food Chemistry, 166, 380-388.

Valencia, Z., Cámara, F., Ccapa, K., Catacora, P., and Quispe, F. (2017). Compuestos bioactivos y actividad antioxidante de semillas de quinua peruana (Chenopodium quinoa W.). Revista de la Sociedad Química del Perú, 83(1), 16-29.

Vidaurre-Ruiz, J. M., Días-Rojas, G., Mendoza-Llamo, E., and Solano-Cornejo, M. Á. (2017). Variación del contenido de Betalaínas, compuestos fenólicos y capacidad antioxidante durante el procesamiento de la quinua (Chenopodium quinoa W.). Revista de la Sociedad Química del Perú, 83(3), 319-329.

von Elbe, J. H. (2001). Current Protocols in Food Analytical Chemistry.

Wang, L., Dong, J., Zhu, Y., Shen, R., Wu, L., and Zhang, K. (2021). Effects of microwave heating, steaming, boiling and baking on the structure and functional properties of quinoa (Chenopodium quinoa Willd.) protein isolates. International Journal of Food Science and Technology, 56(2), 709-720. https://doi.org/10.1111/IJFS.14706 